\title{
ANALIZA ELEMENATA KVALITETA USLUGA U KORPORATIVNOM BANKARSTVU
}

\author{
Veljko Marinković, ${ }^{*}$ Vladimir Senić
}

\begin{abstract}
Fokusiranost na potrošače poslednjih godina doživljava ekspanziju u uslužno orijentisanim delatnostima, uključujući i banke. Potrebe korisnika finansijskih usluga dinamično se menjaju, zbog čega je neophodno da banke razvijaju dugoročne poslovne odnose sa svojim klijentima, kako bi postale i ostale profitabilne. Cilj ovog istraživanja usmeren je na ispitivanje stavova korporativnih klijenata - malih i srednjih preduzeća - o različitim elementima kvaliteta usluga, koje pružaju bankarske institucije. Rezultati realizovane studije ističu tri ključna elementa kvaliteta uslužne ponude u korporativnom bankarstvu: poštovanje i razumevanje klijenata, profesionalnost i imidž. Ovakvi rezultati dodatno potvrđuju da bankarske institucije treba tesno da sarađuju sa klijentima, što je moguće samo ako se istinski posvete marketinškoj orijentaciji u svakodnevnom poslovanju.
\end{abstract}

Ključne reči: kvalitet usluge, imidž banke, mala i srednja preduzeća, korporativni klijenti

\section{JEL Classification: M31}

\section{UVOD}

U protekle dve decenije mala i srednja preduzeća (MSP) privukla su pažnju bankarskih institucija. Ovo ne treba da iznenađuje s obzirom na to da su MSP bitni nosioci savremene ekonomije. Sveobuhvatna studija koju su sproveli Ayyagari, Beck \& Demirgüç-Kunt (2007) ukazala je na to da su MSP u 76 razvijenih zemalja i zemalja u razvoju stvorila preko $50 \%$ radnih mesta u proizvodnji, dok relevantni podaci za 35 zemalja u istoj studiji pokazuju da su MSP u proseku generisala oko $42 \%$ BDP-a. Takođe, po podacima Evropske komisije (2010) u 2008. godini postojao je 21000000 registrovanih

\footnotetext{
* Korespondencija: V. Marinković, Ekonomski fakultet Univerziteta u Kragujevcu, Đ. Pucara 3, 34000 Kragujevac, Srbija; e-mail: vmarinkovic@kg.ac.rs
}

preduzeća u Evropskoj uniji (EU) od čega je svega 43000 bilo velikih preduzeća. Drugim rečima, bilo je 20700000 MSP koja su činila 99,8\% svih preduzeća u EU, i pritom zapošljavala preko $67 \%$ radne snage $u$ EU. Uz to, Shen, Shen, Xu \& Bai (2009) utvrdili su da 99,6\% svih kineskih preduzeća spada u MSP, koja čine skoro $60 \%$ BDP-a i stvaraju tri od četiri radna mesta u urbanim sredinama.

Evidentno, MSP predstavljaju glavnu pokretačku snagu u razvijenim zemljama, kao i u zemljama u razvoju. Ovo je potvrđeno i u studiji Republičkog zavoda za statistiku (2008) objavljenoj 2007. godine. Naime, ukoliko se broj zaposlenih koristi kao glavni kriterijum za utvrđivanje veličine preduzeća, 96,2\% srpskih preduzeća spada u kategoriju mikro i malih preduzeća (do 50 zaposlenih), 3,1\% svrstava se u srednja preduzeća 
(od 50 do 249 zaposlenih) dok preostalih 0,7\% spada u velika preduzeća (više od 250 zaposlenih).

Zastupljenost MSP u svetskoj ekonomiji zapazile su finansijske institucije. Mnoge banke su prepoznale MSP kao potencijalni izvor znatne dobiti (Connolly, 2000). Ovo je još izraženije ako se uzmu u obzir rezultati OEBS-ove (2009) studije, koja pokazuje da se MSP, kada imaju potrebu za dodatnim sredstvima kako bi podržala svoj dalji rast, najčešće okreću pozajmljivanju od banaka, čineći bankarske institucije jednim od najznačajnijih izvora eksternog kapitala za MSP. Pored toga, Torre, Pería \& Schmukler (2010) potvrđuju da mnoge bankarske insititucije prilaze segmentu MSP kao važnom i planiraju da dalje uspešno posluju sa njim pružanjem adekvatnih bankarskih usluga, prilagođenim specifičnim potrebama MSP. Ove konstatacije su u skladu sa onima koje su imali i Silver \& Vegholm (2009). Oni smatraju da bi banke trebalo da utvrde potrebe MSP i prilagode im se kako bi dodatno iskoristile njihov poslovni potencijal.

\section{PREGLED LITERATURE}

\section{Lojalnost kupaca}

Lojalnost kupaca predstavlja jednu od najčešće istraživanih oblasti u marketingu današnjice. Pokazuje zainteresovanost kupaca prema datom preduzeću tokom dužeg vremenskog perioda, istovremeno preporučujući preduzeće i njegove proizvode/usluge drugima. U ranoj fazi istraživanja najviše pažnje je bilo posvećeno ispitivanju lojalnosti brendirane industrijske robe široke potrošnje (Cunningham, 1956) dok je predmet istraživanja lojalnosti u uslugama skoriji fenomen, koji počinje devedesetih godina XX veka. Neki istraživači veruju da je lojalnost još uvek nedovoljno istražena oblast i da nije do sada dobila pažnju koju zaslužuje (Lewis \& Soureli, 2006). Ipak, vrlo brzo je postalo evidentno da je lojalnost od izuzetnog značaja za uslužno orijentisana preduzeća i da bi stoga na lojalnost trebalo gledati kao na najvredniju aktivu za sve ponuđače usluga (Andreassen \& Lindestad, 1998).

Merenje lojalnosti kupaca, međutim, nije lak zadatak (Bauman, Burton \& Eliot, 2005). Složenost prilikom merenja lojalnosti kupaca proizilazi iz činjenice da se koncept lojalnosti sastoji od dimenzije ponašanja i dimenzije stava (Rauyruen \& Miller, 2007). Dimenzija ponašanja se javlja ukoliko kupci kontinuirano kupuju kod istog proizvođača. Kao takva, lojalnost bazirana na ponašanju predstavlja nameru za ponovnom kupovinom usluga/proizvoda - uključujući učestalost i iznos (Lewis \& Soureli, 2006). S druge strane, lojalnost bazirana na stavu podrazumeva kupčevu prirodu, preferencije, poverenje ili emotivne veze ka uslugama/ proizvodima i usmenoj propagandi (Zeithaml, Berry \& Parasuraman, 1996, Andreassen \& Lindestad, 1998). Ipak, treba naglasiti da je gledište po kojem stav i ponašanje čine dve najznačajnije komponente lojalnosti bilo kritikovano u marketinškoj literaturi (Bloemer, Ruyter \& Peeters, 1998).

\section{Kvalitet usluga}

Kvalitet usluga predstavlja jedan od najbitnijih "okidača“ lojalnosti kod kupaca i generiše važan obim interesovanja među istraživačima i praktičarima. Kao termin, kvalitet se koristi često u svakodnevnom životu, i svi imaju dobru ideju o tome šta je dobar, a šta loš kvalitet. Interesantno je da, uprkos tako široko rasprostranjenoj upotrebi, istraživači još uvek nisu uspeli da nađu jedinstvenu definiciju kvaliteta, pre svega zbog toga što je kvalitet, kao koncept, vezan za veliki broj interpretacija (Garvin, 1984). Ono što jedna osoba percipira kao kvalitetno druga osoba može videti kao prosečno ili ispodprosečno. Naime, elementi koji uključuju navike, stavove, iskustva, set vrednosti ili društvenu klasu pojedinca, samo su neki faktori koji utvrđuju način na koji osoba može percipirati kvalitet. Ovo jasno sugeriše da je merenje kvaliteta složen i izazovan zadatak. Kompleksnost merenja kvaliteta je dodatno produbljena nekim karakteristikama usluga, uključujući neopipljivost, ali i činjenicom da su kupci često uključeni u uslužni proces i da samim tim direktno utiču na kvalitet usluge.

Složenost kod merenja kvaliteta u uslugama najbolje ilustruju Seth, Deshmukh \& Vrat (2005) koji su u svojoj studiji predstavili 19 različitih modela, koji se koriste za merenje kvaliteta usluge. Tehnički i funkcionalni kvalitet (Grönroos, 1984), model gepova (Parasuraman, Zeithaml \& Berry, 1985), model baziran isključivo na 
performansama (Cronin \& Taylor, 1992) i hijerarhijski model (Brady \& Cronin, 2001) predstavljaju najznačajnije modele, koji se često koriste u savremenim istraživanjima. Jedan od najčešće korišćenih modela za merenje kvaliteta usluge je SERVQUAL (Parasuraman, Zeithaml \& Berry, 1988), koji je korišćen prilikom sastavljanja upitnika za ovo istraživanje.

\section{CRM u savremenom bankarstvu}

Oštra konkurencija na tržištu finansijskih usluga primorala je mnoge banke da prihvate načela CRM (upravljanje odnosima sa potrošačima - Customer Relationship Management) koncepta. Danas su banke orijentisane na tesnu poslovnu saradnju sa klijentima, jer njihov opstanak i dalji razvoj često zavise od pružanja dodatnih vrednosti, koje bi trebalo da prevazilaze očekivanja klijenata tokom dužeg perioda. Osim toga, dodatnu vrednost konkurenti ne mogu lako kopirati, zbog čega ona predstavlja važnu determinantu stvaranja održive konkurentske prednosti. U uslovima dinamične tržišne utakmice nije dovoljno da banke budu fokusirane samo na kreiranje kvalitetnih proizvoda i usluga, već je od suštinskog značaja da se izgrade čvrste poslovne veze sa klijentima, a CRM koncept stavlja klijente u centar ukupne bankarske aktivnosti. Međutim, da bi se to postiglo, važno je pretvoriti transakcije u međuljudske relacije, čineći CRM koncept sastavnim i neizostavnim delom savremenog marketinga banke. Implementacija koncepta ,jedan-na-jedan marketinga“ jeste strategija koja omogućava dugoročnu saradnju između banke i svakog klijenta pojedinačno. Ovo predstavlja poseban izazov, naročito za velike i finansijski jake banke, koje pri tome imaju i veliki broj klijenata.

Primena CRM-a je veoma značajna, jer istraživanja pokazuju da su korisnici usluga banaka dinamičniji i sve manje lojalni. Zahvaljujući Internetu, klijentima je danas, više nego ikada ranije, pružena mogućnost prelaska iz jedne banke $\mathrm{u}$ drugu banku (Gupta \& Shukla, 2002). Odnos između banke i klijenata ne predstavlja samo prost zbir realizovanih transakcija u prošlosti (Parasuraman, Zeithaml \& Berry, 1988). Snažna povezanost između dve strane $u$ sektoru finansijskih usluga zasnovana je prvenstveno na obostranoj dugoročnoj koristi. U tom kontekstu, banke kao ponuđači finansijskih usluga planiraju da ostvare maksimizaciju profita povećanjem stepena satisfakcije i lojalnosti klijenata. S druge strane, dugoročnom saradnjom sa određenom bankom, klijenti očekuju da dobiju povoljnije kamate na kredite, bolji kvalitet usluga, kao i da steknu viši nivo poverenja u banku (Binks \& Ennew, 1997). Prihvatanje marketing orijentacije predstavlja odgovor banaka na dejstvo različitih faktora iz poslovnog okruženja. Neophodno je da banke dobro poznaju svoje klijente, kako bi bile u stanju da procene rizik prilikom odobravanja kredita ili obavljanja nekog drugog posla. Kako sugeriše Buttle (2004), dosledna primena marketing koncepta pomaže bankama ne samo da privuku nove, nego i da zadrže postojeće klijente. Marketinški orijentisane banke $u$ fokusu svog poslovanja svakodnevno imaju sadašnje i buduće potrebe klijenata. Uspostavljanje aktivnih poslovnih odnosa i tesnih poslovnih veza sa klijentima preduslovi su stvaranja lojalnosti i dugoročne profitabilnosti banke.

Menadžment mnogih banaka daje prednost i veći značaj korporativnim klijentima u odnosu na klijente koji dolaze iz reda stanovništva. Iako su manje zastupljeni na tržištu, korporativni klijenti imaju složenije finansijske zahteve. Finalni potrošači najčešće biraju banku na osnovu visine kamatnih stopa na oročenu štednju, povoljnosti različitih vrsta kredita, raznovrsnosti platnih kartica koje se nalaze u ponudi banke. Na ovom polju male banke teže mogu parirati velikimbankama.Za razlikuod tržištafinalnepotrošnje, na poslovnim tržištima lakše se vide prednosti malih banaka. Bez obzira na činjenicu da manje banke možda nemaju mogućnost da u potpunosti zadovolje finansijske potrebe velikih kompanija, one mogu veoma uspešno izaći $u$ susret malim preduzećima. Poštovanje i razumevanje klijenata predstavljaju značajne aspekte za uspostavljanje dugoročne saradnje između malih banaka i malih i srednjih preduzeća. Pronalaženje adekvatne poslovne niše jeste osnova za poboljšanje konkurentske pozicije male banke. Da bi se to ostvarilo, banka mora ispuniti dva važna uslova prvi je orijentisanost na klijente, a drugi posedovanje moderne informaciono-komunikacione tehnologije.

Faktori koji imaju presudan uticaj na intenzitet poslovnih odnosa između banaka i korporativnih klijenata su nivo konkurencije $u$ grani, veličina 
preduzeća, potreba za uzimanjem kredita ili korišćenjem neke druge bankarske usluge, troškovi obavljanja transakcija i finansijska situacija preduzeća. Generalno, velika preduzeća imaju snažniju potrebu da razvijaju poslovne odnose sa većim brojem banaka od malih preduzeća. Takođe, veličina kompanije nekad uslovljava i njenu pregovaračku poziciju. Velike kompanije manje zavise od poslovnih odnosa koje uspostavljaju sa određenom bankom, s obzirom na velike mogućnosti saradnje i sa ostalim finansijskim institucijama. S druge strane, kako Zineldin (1996) naglašava, mala preduzeća imaju veći motiv za uspostavljanje dobrih dugoročnih poslovnih odnosa sa bankom kojoj žele da budu lojalni klijenti.

Ekspanzija Interneta i savremenih informacionokomunikacionih tehnologija omogućila je i razvoj elektronskog bankarstva. Sada se interakcija između bankara i klijenta ne zasniva samo na personalnim vezama i kontaktima, nego i na elektronskim odnosima koje podržava informaciono-komunikaciona tehnologija, što omogućava bržu i precizniju realizaciju transakcija. Podaci koji pokazuju rast u korišćenju usluga elektronskog bankarstva su sledeći: procenjuje se da je tokom 2000. godine oko 34400000 ljudi koristilo usluge elektronskog bankarstva. Do 2004. godine ovaj broj se povećao na 122300000 . Finska je tipičan primer zemlje za čije građane elektronsko bankarstvo zaista predstavlja izvor dodatne vrednosti. Prema podacima OEBS-a, u aprilu 2005. godine, oko 2600000 Finaca (polovina od ukupne populacije Finske) koristilo je usluge elektronskog bankarstva (Maenpaa, Kale \& Mesiranta, 2008). Svega tri posto Finaca plaća, ili vrši druge transakcije, u samoj filijali. Međutim, iako se primena CRM-a ne može zamisliti bez moderne informacione tehnologije, menadžeri ne smeju zanemariti važnost ličnih odnosa, jer oni predstavljaju temelj dugoročne saradnje. Banke, pre svega, moraju pokazati da razumeju i poštuju svoje klijente, da brinu o njima i da su iskreno usmerene na rešavanje njihovih problema i finansijskih potreba.

\section{MSP kao korporativni klijenti bankarskog sektora}

U svim visokokonkurentnim sektorima, uključujući bankarstvo, važno je stalno pružanje kvalitetne usluge. U svojoj studiji, Wang, Lo \& Hui (2003) ukazuju na to da su kineske banke, koje pružaju usluge vrhunskog kvaliteta svojim korporativnim klijentima, stekle reputaciju da imaju izuzetnu uslugu, što je dovelo do višeg stepena zadržavanja korporativnih klijenata. Sporedni efekat je to što zadovoljni korporativni klijenti nisu želeli da posluju sa konkurentskim bankama. Istraživanja o lojalnosti u bankarskom sektoru često su obavljana u marketinškoj literaturi (Lewis \& Soureli, 2006; Berg, 2008). Veliki broj studija o lojalnosti i faktorima, koji vode ka lojalnosti u uslužnom sektoru, evoluirao je iz ispitivanja sprovedenih nad stanovništvom koje posluje sa bankama (Hallowell, 1996). Međutim, svega nekoliko istraživanja bilo je sprovedeno $\mathrm{u}$ kontekstu ispitivanja lojalnosti među korporativnim klijentima banaka (Lam, Burton \& Lo, 2009) iako oni generišu veliki deo prihoda bankarskih institucija (Tyler \& Stanley, 1999).

Jedan broj studija bio je fokusiran na utvrđivanje faktora koji utiču na korporativne klijente prilikom odabira banke. Faktori vezani za spektar ponuđenih usluga, cene usluga, personalizovanu interakciju, pristupačnost i interesne stope kreditnih linija, istakli su se kao najznačajniji za preduzeća prilikom njihovog odabira banke (Nielsen, Terry \& Trayler, 1998). Istraživanja su, takođe, sugerisala da tehnički i funkcionalni kvalitet usluga imaju pozitivan efekat na lojalnost MSP, naglašavajući pritom da ulaganje $u$ stvaranje snažnih odnosa sa MSP donosi veću lojalnost preduzeća (Madill, Feeney, Riding \& Haines Jr, 2002). S druge strane, Lam \& Burton (2009) su bili među prvima koji su kvantifikovali uticaj koji različite varijable imaju na lojalnost MSP. Njihovo istraživanje pokazalo je da, ukoliko žele da maksimiziraju nivoe lojalnosti među MSP, banke treba da pruže kvalitetnu uslugu, ulažući vreme u negovanje odnosa klijent-banka i $u$ kreiranje cenovnih strategija za kredite ponuđene MSP.

Konačno, u svojoj studiji Silver \& Vegholm (2009) bavili su se pitanjem vezanim za spremnost banaka da se prilagode svojim MSP klijentima. Ovo istraživanje pokazalo je da se banke ne prilagođavaju mnogo svojim MSP klijentima, pre svega zbog centralizovane organizacione strukture banaka, nedovoljne komunikacije sa MSP i nedostatka razumevanja potreba MSP. Nespremnost banaka da se prilagode potrebama MSP dodatno je naglašena kada su u pitanju napori 
MSP da internacionalizuju svoje poslovanje. Iako je već utvrđeno da su MSP zavisna od bankarskih/ finansijskih institucija prilikom širenja na strana tržišta (Spence, 2003), nedavna studija, koju su sproveli Lindstrad \& Lindbergh (2010), otkriva da banke loše koriste svoju ulogu mogućeg ponuđača informacija i usluga onim MSP koja se internacionalizuju.

\section{METODOLOGIJA ISTRAŽIVANJA}

Da bi se ispitivao nivo zadovoljstva klijenata različitim elementima uslužne ponude banaka, sprovedeno je empirijsko istraživanje primenom anketnog metoda. Anketni metod je jedan od najčešć korišćenih metoda $\mathrm{u}$ terenskim marketinškim istraživanjima. Upitnik kojim su prikupljeni primarni podaci obuhvatio je 21 konstataciju koje odražavaju različite elemente kvaliteta usluga u bankarstvu. Ispitanici su stepen slaganja sa navedenim konstatacijama iskazivali na desetostepenoj skali (1 - apsolutno se ne slažem sa konstatacijom; 10 - apsolutno se slažem sa konstatacijom). Konstatacije su odabrane na osnovu pregleda relevantne literature iz oblasti marketinga usluga (Parasuraman, Zeithaml \& Berry, 1988; Jamal \& Anastasiadou, 2009). Time je stvorena osnova za analizu ljubaznosti, profesionalnosti i kompetentnosti zaposlenih službenika na osnovu percepcija klijenata. Osim toga, analiziran je i imidž banke, kao i stepen saosećanja službenika sa problemima klijenata.

U istraživanje su uključena 133 preduzeća, čiji su menadžeri ocenili kvalitet usluga banke preko koje realizuju poslovne transakcije. Reč je o malim i srednjim preduzećima koja imaju do 250 zaposlenih. Najveći broj korporativnih klijenata banaka, koji posluju na teritoriji Srbije, upravo spada $\mathrm{u}$ grupu malih i srednjih preduzeća. Uz to, ova preduzeća su i značajni nosioci razvoja nacionalne ekonomije. $U$ tom kontekstu, studija je usmerena upravo ka ispitivanju stavova menadžera malih i srednjih preduzeća o kvalitetu usluga $u$ bankarstvu. Korporativni klijenti su uključeni u uzorak metodom slučajnog izbora, a kao okvir za izvlačenje jedinica poslužila je lista korporativnih klijenata date banke $\mathrm{u}$ prethodnom petogodišnjem periodu. Pri tome, svi klijenti su razvrstani u tri segmenta (veliki, srednji i mali klijenti) na osnovu visine godišnjeg prometa, koji realizuju preko banke. Prvi segment (veliki klijenti) obuhvatio je ukupno 30 preduzeća, drugi segment (srednji klijenti) 48 preduzeća dok 55 preduzeća spada u grupu malih klijenata. Pre same distribucije upitnik je testiran na manjem uzorku od 15 ispitanika kako bi se uklonilo sve što je eventualno nejasno $u$ vezi sa upitnikom. Osim toga, da bi se kreirao jasan i pregledan upitnik, istraživači su organizovali i jednu grupnu diskusiju sa bankarskim službenicima.

Analiza podataka sprovedena je u statističkom paketu za društvene nauke (The Statistical Package for the Social Sciences - SPSS). Od statistističkih analiza implementirane su eksplorativna faktorska analiza i analiza varijanse. Primenom faktorske analize veliki broj konstatacija grupisan je u manji broj faktora. Pouzdanost dobijenih faktora i interna konzistentnost tvrdnji mereni su preko vrednosti koeficijenta alfa. Primenom Kajzer-Mejer-Olkinovog (KMO) testa i Bartletovog testa ispitana je prikladnost podataka za sprovođenje faktorske analize. Testiranje jednakosti prosečnih vrednosti konstatacija za tri grupe klijenata urađeno je pomoću one-way ANOVA-e. Primena navedenog postupka predstavlja logično rešenje za poređenje sredina u situacijama kada postoji više od dve grupe ispitanika.

\section{REZULTATI ISTRAŽIVANJA}

Pre same implementacije faktorske analize, vrednosti KMO testa $(\mathrm{KMO}=0,877)$ i Bartletovog testa $(\mathrm{p}=0,000)$ potvrdile su da postoje opravdani uslovi za njenu primenu. Vrednost KMO indeksa može se kretati $u$ intervalu između 0 i 1, a korišćenje faktorske analize je neadekvatno ukoliko su KMO vrednosti ispod 0,5. Bartletov test baziran je na hi-kvadrat statistici. Dobijena vrednost pokazuje da se odbacuje nulta hipoteza (nepostojanje značajne korelacije između promenljivih). Kao metod faktorske analize u ovom istraživanju korišćena je analiza glavnih komponenata, a zbog jasnije interpretacije faktora, upotrebljena je varimaks rotacija.

Rezultati faktorske analize pokazuju da su se konstatacije grupisale oko tri formirana faktora. 
Prvi faktor "Poštovanje i razumevanje klijenata“ opisuje 31,776\% varijanse. Ovaj faktor obuhvata atribute kvaliteta usluge banaka koji se odnose na posvećivanje lične pažnje klijentima, ljubaznost službenika, razumevanje finansijskih potreba klijenata, spremnost da se pomogne klijentima i sl. Drugi faktor „Profesionalnost“ objašnjava 19,033\% varijanse. Reč je o faktoru koji uključuje uslužne elemente kao što su preciznost i brzina pružene usluge, ispunjenje obećanja i poštovanje dogovorenih rokova od strane službenika. Treći faktor "Imidž“ kao svoje osnovne elemente sadrži imidž banke, imidž filijale, savremenu opremu, enterijer, kao i imidž u poređenju sa imidžom konkurenata. Dati faktor pridružuje 15,892\% varijanse. Inače, sva tri faktora opisuju 66,701\% ukupne varijanse.

Sva tri faktora imaju visok nivo pouzdanosti. Kao što se u Tabeli 1 može videti, vrednosti alfa koeficijenta

Tabela 1 Rezultati eksplorativne faktorske analize

\begin{tabular}{|c|c|c|c|}
\hline Konstatacije & Faktor 1 & Faktor 2 & Faktor 3 \\
\hline \multicolumn{4}{|l|}{ Faktor 1: Poštovanje i razumevanje klijenata } \\
\hline Posvećivanje lične pažnje klijentima & 0,831 & & \\
\hline Ljubaznost zaposlenih službenika & 0,817 & & \\
\hline Poštovanje klijenata & 0,798 & & \\
\hline Razumevanje finansijskih potreba klijenata & 0,784 & & \\
\hline Fokus ka onome što je najbolje za klijente & 0,748 & & \\
\hline Spremnost da se pomogne klijentu & 0,741 & & \\
\hline Poverenje klijenata u službenike banke & 0,692 & & \\
\hline Orijentacija na klijente & 0,648 & & \\
\hline Osećaj sigurnosti kod klijenata & 0,619 & & \\
\hline Pružanje informacija klijentima & 0,590 & & \\
\hline \multicolumn{4}{|l|}{ Faktor 2: Profesionalnost } \\
\hline Preciznost pružene usluge & & 0,707 & \\
\hline Brzina pružanja usluge & & 0,694 & \\
\hline Izgled zaposlenih & & 0,669 & \\
\hline Učinjeni napori ka rešavanju problema klijenata & & 0,654 & \\
\hline Poštovanje dogovorenih rokova sa klijentom & & 0,648 & \\
\hline Ispunjenje obećanja datih klijentima & & 0,558 & \\
\hline \multicolumn{4}{|l|}{ Faktor 3: Imidž } \\
\hline Imidž u odnosu na konkurente & & & 0,828 \\
\hline Enterijer & & & 0,820 \\
\hline Savremena oprema & & & 0,803 \\
\hline Imidž banke & & & 0,663 \\
\hline Imidž filijale & & & 0,618 \\
\hline Eigenvalue & 6,673 & 3,997 & 3,337 \\
\hline Procenat opisane varijanse & 31,776 & 19,033 & 15,892 \\
\hline Alfa & 0,94 & 0,82 & 0,84 \\
\hline
\end{tabular}


su više od minimalno potrebnog praga pouzdanosti od 0,7, koji preporučuje Nunnally (1978). Budući da su dobijene visoke vrednosti alfa koeficijenta, koje su kod sva tri faktora više od 0,8, može se zaključiti da su konstatacije koje su se grupisale oko svakog pojedinačnog faktora interno konzistentne.

Primenom ANOVA testa dobijeni su veoma interesantni rezultati poređenja sredina između korporativnih klijenata, koji pripadaju različitim segmentima (Tabela 2). Naime, na osnovu visine godišnjeg prometa koji svako preduzeće realizuje preko date banke, formirana

Tabela 2 Rezultati ANOVA testa

\begin{tabular}{|c|c|c|}
\hline Konstatacije & $\mathrm{F}$ & Značajnost \\
\hline Posvećivanje lične pažnje klijentima & 0,675 & 0,511 \\
\hline Ljubaznost zaposlenih službenika & 0,543 & 0,582 \\
\hline Poštovanje klijenata & 0,910 & 0,405 \\
\hline $\begin{array}{l}\text { Razumevanje finansijskih potreba } \\
\text { klijenata }\end{array}$ & 0,841 & 0,434 \\
\hline $\begin{array}{l}\text { Fokus ka onome što je najbolje za } \\
\text { klijente }\end{array}$ & 0,159 & 0,853 \\
\hline Spremnost da se pomogne klijentu & 0,155 & 0,857 \\
\hline Poverenje klijenata u službenike banke & 0,762 & 0,469 \\
\hline Orijentacija na klijente & 0,733 & 0,482 \\
\hline Osećaj sigurnosti kod klijenata & 0,243 & 0,785 \\
\hline Pružanje informacija klijentima & 0,243 & 0,785 \\
\hline Preciznost pružene usluge & 0,928 & 0,398 \\
\hline Brzina pružanja usluge & 1,596 & 0,207 \\
\hline Izgled zaposlenih & 1,049 & 0,353 \\
\hline $\begin{array}{l}\text { Učinjeni napori ka rešavanju problema } \\
\text { klijenata }\end{array}$ & $2,969^{*}$ & 0,045 \\
\hline $\begin{array}{l}\text { Poštovanje dogovorenih rokova sa } \\
\text { klijentom }\end{array}$ & $5,654^{* *}$ & 0,004 \\
\hline Ispunjenje obećanja datih klijentima & 0,762 & 0,469 \\
\hline Imidž u odnosu na konkurente & 0,483 & 0,618 \\
\hline Enterijer & 0,602 & 0,549 \\
\hline Savremena oprema & 1,616 & 0,203 \\
\hline Imidž banke & 0,016 & 0,984 \\
\hline Imidž filijale & 0,133 & 0,875 \\
\hline
\end{tabular}

$* * p<0,01 ; \quad * p<0,05$ su tri segmenta: veliki, srednji i mali klijenti. Na osnovu svake konstatacije utvrđeno je da li se javljaju statistički značajne razlike $\mathrm{u}$ percepcijama klijenata. Međutim, dobijeni rezultati naglašavaju ujednačenost stavova korporativnih klijenata o različitim elementima kvaliteta uslužne ponude banke. Samo u slučaju konstatacije „Učinjeni napori ka rešavanju problema klijenata" i konstatacije „Poštovanje dogovorenih rokova sa klijentom" ispoljile su se razlike u sredinama između posmatrane tri grupe ispitanika. U ostalim konstatacijama srednje vrednosti u posmatranim grupama su jednake.

\section{ZAKLJUČAK}

Savremeni trendovi $\mathrm{u}$ bankarskom poslovanju naglašavaju sve veću važnost ličnih interakcija između bankara i klijenata za dalji razvoj njihovih dugoročnih poslovnih odnosa. Ukoliko banka želi da sa određenim klijentom razvija dugoročne poslovne odnose, veoma je važno da se procene budući novčani tokovi koje će banka ostvariti saradnjom sa datim klijentom. Međutim, da bi se stekla jasna slika o očekivanim događajima, imperativ je da banka bude marketinški orijentisana.

Dobar odnos sa klijentima važan je faktor ne samo za poboljšanje konkurentnosti banke, već i za postizanje odgovarajućih poslovnih ciljeva u dinamičnoj tržišnoj utakmici. Uspostavljanje tešnjih poslovnih odnosa sa klijentima trebalo bi da bude svakodnevni cilj koji se ostvaruje pokazivanjem pažnje, poštovanjem, razumevanjem i empatijom prema klijentima, $s$ jedne strane, odnosno brzinom i preciznošću prilikom obavljanja transakcija, $\mathrm{s}$ druge strane. Uspešna komunikacija sa klijentom, kompetentni kadrovi i moderna informaciono-komunikaciona tehnologija predstavljaju tri ključna elementa marketinške strategije banke. Bez adekvatne podrške finansijskog sektora, marketing sam po sebi ne može pomoći banci da ostvari dugoročnu profitabilnost. Menadžeri moraju biti svesni činjenice da se klijenti iz reda stanovništva upravljaju prema visini kamatnih stopa na oročenu štednju ili prema povoljnosti kredita, dok korporativni klijenti biraju banku primarno na osnovu sposobnosti banke da podmiri njihove finansijske potrebe. 
Nalazi empirijskog istraživanja ističu važnost tri elementa uslužne ponude banaka: poštovanje i razumevanje klijenata, profesionalnost zaposlenih službenika i imidž banke. Prva dva faktora odražavaju neopipljive elemente uslužne ponude banaka i oni ukazuju na značaj unapređenja interakcija sa klijentima, kojepodrazumevaju poštovanje, razumevanjeproblema klijenata, empatiju, kao i ažurnu i preciznu realizaciju finansijskih transakcija. Imidž, kao treći identifikovani faktor, uključuje i opipljive elemente kvaliteta, kao što su moderna oprema i uređenost enterijera $u$ filijalama. Rezultati implementirane studije ukazuju i na to da je konkretna banka, čiji su korporativni klijenti iz redova malih i srednjih preduzeća anketirani, uspela da uspostavi sličan stepen kvaliteta odnosa sa različitim klijentima, bez obzira na vrednost $\mathrm{i}$ učestalost transakcija koje oni obavljaju preko date banke. Naime, signifikantne statističke razlike $u$ ocenama kljenata iz različitih segmenata pojavile su se samo na osnovu dva ispitivana atributa.

Važno je istaći da je sprovedena studija bila usmerena pre svega na merenje kvaliteta usluga u korporativnom bankarstvu. U budućim istraživanjima korisno bi bilo da se analizom obuhvate i finansijski elementi, kao što su visina kamatnih stopa, raznovrsnost platnih kartica i povoljnosti korišćenja kredita. Bilo bi interesantno utvrditi da li su, i u kojoj meri, navedeni elementi značajniji za klijentov izbor banke u odnosu na neopipljive uslužne elemente. Osim toga, korisno je da se sprovede komparativna analiza stavova i percepcija korporativnih klijenata i klijenata iz reda stanovništva.

Buduća istraživanja mogla bi i da ispitaju uticaj koji faktori uslužne ponude banaka imaju na lojalnost klijenata. U tom kontekstu korisno je da se koncipira i testira novi model, koji bi obuhvatio tri nezavisne varijable (poštovanje i razumevanje klijenata, profesionalnost i imidž) i jednu zavisnu varijablu (lojalnost klijenata). Primenom višestruke regresione analize, može se utvrditi statistička značajnost uticaja navedenih faktora na lojalnost klijenata. Pored toga, implementacijom moderacijske regresije mogu se, osim glavnih efekata, takođe testirati i interakcijski efekti. Time bi se dobila sveobuhvatnija analiza ključnih antecedenti lojalnosti u korporativnom bankarstvu.

\section{ZAHVALNICA}

Ovaj rad je deo interdisciplinarnog istraživačkog Projekta (broj 41010), koji finansira Ministarstvo nauke Republike Srbije.

\section{REFERENCE}

Andreassen, T., \& Lindestad, B. (1998). Customer loyalty and complex services. International Journal of Service Industry Management, 9 (1), 7-23.

Ayyagari, M., Beck, T., \& Demirgüç-Kunt, A. (2007). Small and medium enterprises across the globe. Small Business Economics, 29 (4), 415-434.

Berg, L. (2008). Loyalty, naivety and powerlessness among Norwegian retail bank customers. International Journal of Consumer Studies, 32 (3), 222-232.

Binks, M. R., \& Ennew, C. T. (1997). The Relationship Between UK Banks and Their Small Business Customers. Small Business Economics, 9 (2), 167-178.

Bloemer, J., Ruyter, K., \& Peeters, P. (1998). Investigating drivers of bank loyalty: the complex relationship between image, service quality and satisfaction. International Journal of Bank Marketing, 16 (7), 276-286.

Brady, M. K., \& Cronin Jr, J. J. (2001). Some new thoughts on conceptualizing perceived service quality: a hierarchical approach. Journal of Marketing, 65 (3), 34-49.

Buttle, F. (2004). Customer Relationship Management. Amsterdam: Elsevier.

Connolly, B. J. (2000). Banks must manage small business deposits to foster long-term growth. Commercial Lending Review, 15 (4), 8-14.

Cronin, J. J., \& Taylor, A. S. (1992). Measuring service quality: a reexamination and an extension. Journal of Marketing, 56 (3), 55-67.

Cunningham, R. M. (1956). Brand loyalty - what, where, how much. Harvard Business Review, 39 (11/12), 116-138.

Evropska Komisija, Generalni direktorat za preduzetništvo $\mathrm{i}$ industriju (2010). European SMEs under pressure: annual report on EU small and medium-sized enterprises 2009. Brussels: European Commission.

Garvin, D. (1984). What does 'product quality' really mean? Sloan Management Review, 26 (1), 25-43. 
Grönroos, C. (1984). A service quality model and its marketing implications. European Journal of Marketing, 18 (4), 244-249.

Gupta, M. P., \& Shukla, S. (2002). Learning from Customer Relationship Management Implementation in a Bank. Global Business Review, 3 (1), 99-122.

Hallowell, R. (1996). The relationships of customer satisfaction, customer loyalty, and profitability: an empirical study. International Journal of Service Industry Management, 7 (4), 2742.

Jamal, A., \& Anastasiadou, K. (2009). Investigating the effects of service quality dimensions and expertise on loyalty. European Journal of Marketing, 43 (3/4), 398-420.

Lam, R., Burton, S., \& Lo, H.-P. (2009). Customer tradeoffs between key determinants of SME banking loyalty. International Journal of Bank Marketing, 27 (6), 428-445.

Lewis, B. R., \& Soureli, M. (2006). The antecedents of consumer loyalty in retail banking. Journal of Consumer Behaviour, 5 (1), 15-31.

Lindstrand, A., \& Lindbergh, J. (2011). SMEs' dependency on banks during international expansion. International Journal of Bank Marketing, 29 (1), 65-83.

Madill, J.J., Feeney, L., Riding, A., \& Haines Jr, G. H. (2002). Determinants of SME owners' satisfaction with their banking relationships: a Canadian study. International Journal of Bank Marketing, 20 (2), 86-98.

Maenpaa, K., Kale, S., Kuusela, H., \& Mesiranta, N. (2008). Consumer perceptions of Internet banking in Finland: The moderating role of familiarity. Journal of Retailing and Consumer Services, 15 (4), 266-276.

Nielsen, J.F., Terry, C., \& Trayler, R.M. (1998). Business banking in Australia: a comparison of expectations. International Journal of Bank Marketing, 16 (6), 253-263.

Nunnally, J.C. (1978). Introduction to psychological measurement. New York, NY: McGraw-Hill.

OEBS (2009). The impact of the global crisis on SME and entrepreneurship financing and policy response. Paris: OECD.

Parasuraman, A., Zeithaml, V. A., \& Berry, L. L. (1985). A conceptual model of service quality and its implications for future research. Journal of Marketing, 49 (4), 41-50.
Parasuraman, A., Zeithaml, V. A., \& Berry, L. L. (1988). SERVQUAL: a multiple-item scale for measuring consumer perception of service quality. Journal of Retailing, 64 (1), 1240 .

Rauyruen, P., \& Miller, K. E. (2007). Relationship quality as a predictor of B2B customer loyalty. Journal of Business Research, 60 (1), 21-31.

Republički zavod za statistiku (2008). Enterprises in Republic of Serbia according to size: vol. 61, Belgrade: Statistical Office (in Serbian). Available: http://webrsz.stat.gov.rs/axd/ dokumenti/rdokument. 16. nov. 2008.

Seth, N., Deshmukh, S. G., \& Vrat, P. (2005). Service quality models: a review. International Journal of Quality and Reliability Management, 22 (9), 913-949.

Shen, Y., Shen, M., Xu, Z., \& Bai, Y. (2009). Bank size and small and medium-sized enterprise (SME) lending: evidence from China. World Development, 37 (4), 800-811.

Silver, L., \& Vegholm, F. (2009). The dyadic bank-SME relationship: customer adoption in interaction, role and organization. Journal of Small Business and Enterprise Development, 16 (4), 615-627.

Spence, M. (2003). International strategy formation in small Canadian high-technology companies - a case study approach. Journal of International Entrepreneurship, 1 (3), 27796.

Torre, A. de la, Martínez Pería, M. S., \& Schmukler, S. L. (2010). Bank involvement with SMEs: beyond relationship lending. Journal of Banking and Finance, 34 (9), 2280-2293.

Tyler, K., \& Stanley, E. (1999). Marketing financial services to businesses: a critical review and research agenda. International Journal of Bank Marketing, 17 (3), 98-115.

Wang, Y., Lo, H.P., \& Hui, Y.V. (2003). The antecedents of service quality and product quality and their influences on bank reputation: evidence from the banking industry in China. Managing Service Quality, 13 (1), 72-83.

Zeithaml, V. A., Berry, L. L., \& Parasuraman, A. (1996). The behavioral consequences of service quality. Journal of Marketing, 60 (2), 31-46.

Zineldin, M. (1996). Bank-corporate client "partnership" relationship: benefits and life cycle. International Journal of Bank Marketing, 14 (3), 14-22. 
Veljko Marinković je docent Ekonomskog fakulteta Univerziteta u Kragujevcu, na nastavnim predmetima Ponašanje potrošača i Istraživanje tržišta. Doktorirao je na Ekonomskom fakultetu Univerziteta u Beogradu. Oblasti njegovog naučnog interesovanja su merenje satisfakcije i lojalnosti potrošača, analiza profitabilnosti potrošača, marketing finansijskih usluga, potrošački etnocentrizam.

Vladimir Senić je docent Fakulteta za hotelijerstvo i turizam Univerziteta u Kragujevcu, na nastavnim predmetima Menadžment usluga i Marketing menadžment. Doktorirao je na Ekonomskom fakultetu Univerziteta u Kragujevcu. Predmet njegovog naučnog i stručnog interesovanja vezan je za problematiku u domenu menadžmenta i marketinga uslužnih organizacija. 


\title{
ANALYSIS OF SERVICE QUALITY ELEMENTS IN CORPORATE BANKING
}

\author{
Veljko Marinkovic, ${ }^{*}$ Vladimir Senic
}

\begin{abstract}
Customer orientation in the last couple of years has been experiencing an expansion in many service-based organizations including banks. The needs of financial services users are dynamically changing, making it necessary for banks to be oriented towards developing long-term relationships with their clients in order to become and remain profitable. The main objective of this research is aimed at studying attitudes of corporate clients - small and medium enterprises - on different elements of the service quality delivered by a banking institution. The results of the conducted study emphasize three key elements of a service offer in corporate banking, including, respecting and understanding a bank's clients, professionalism and image. Such findings additionally confirm the necessity for modern banking institutions to embrace the concept of tight cooperation with clients, only possible if they are truly committed to the marketing orientation in their everyday business.
\end{abstract}

Keywords: service quality, bank image, small and medium enterprises, corporate clients

\section{JEL Classification: M31}

\section{INTRODUCTION}

Over the past two decades, Small and Medium Enterprises (SMEs) have generated a lot of attention on behalf of banking institutions. This should not be much of a surprise considering that SMEs are one of the most significant drivers of the modern economy. An extensive study conducted by Ayyagari, Beck \& Demirgüç-Kunt (2007) found that in 76 developed and developing countries, SMEs contributed over $50 \%$ to employment in manufacturing, while the relevant data for 35 countries in the same study imply that, on

\footnotetext{
* Correspondence to: V. Marinkovic, Faculty of Economics University of Kragujevac, Dj. Pucara 3, 34000 Kragujevac, Serbia; e-mail: vmarinkovic@kg.ac.rs
}

average, SMEs generated about $42 \%$ of the national GDP. Additionally, according to the European Commission (2010) as of 2008, there were almost 21 million registered enterprises within the European Union (EU), of which only 43,000 were large scale enterprises (LSEs). In other words, there were 20.7 million SMEs accounting for $99.8 \%$ of all EU enterprises and employed over $67 \%$ of the EU workforce. Furthermore, Shen, Shen, Xu \& Bai (2009) reported that $99.6 \%$ of all Chinese enterprises were SMEs accounting for almost $60 \%$ of the nation's GDP, while generating 3 out of 4 jobs in urban areas.

Evidently, SMEs represent the major impetus in developed market economies as well as in developing countries. This is confirmed by a study conducted in 2007 by the Statistical Office of the Republic of 
Serbia (2008). Namely, if the number of employees is used as the main criterion for determining the size of enterprises, some $96.2 \%$ of Serbian companies fall into the category of micro and small enterprises (up to 50 employees), $3.1 \%$ are classified as medium enterprises (from 50 to 249 employees), while the remaining 0.7\% belong to large enterprises (more than 250 employees).

Such an evident presence of SMEs in the world's economy has not gone unnoticed by financial institutions. Certainly, many banks have recognized SMEs as a potential source of significant profits (Connolly, 2000). This is even more pronounced considering the results of the OECD (2009) study suggesting that, when in need of additional funds to support further growth, SMEs most frequently turn to bank lending - thus rendering banking institutions as one of the most significant sources of external capital for SMEs. Furthermore, Torre, Pería \& Schmukler (2010) confirm that numerous banking institutions are approaching SME segment as a significant one and do have plans to further increase their presence in this segment through providing adequate banking services tailored to the specific SME needs. Such observations go along with those made by Silver \& Vegholm (2009), who suggest that SMEs need to be treated as clients of great significance, implying that banks should make a substantial effort to identify SMEs' needs and adapt to them accordingly in order to further utilize the business potential that this segment offers.

\section{LITERATURE REVIEW}

\section{Customer loyalty}

Customer loyalty represents one of the most frequently researched areas in marketing nowadays. As a concept, it is used to describe the willingness of customers to continue to show interest in a given company over a longer period of time, while simultaneously recommending the company and its products/services to others. In the early phase of research, most of the attention was dedicated to examining loyalty with respect to branded industrial consumer goods (Cunningham, 1956), while the focus on investigating loyalty in the service sector context is a rather recent phenomenon, truly commencing as early as in the 1990s. Some academics believe that loyalty in service is still a relatively under-researched field and that it has not received the attention it deserves (Lewis \& Soureli, 2006). Nevertheless, it has become apparent fairly quickly that loyalty is of great importance to serviceoriented companies and hence it should be viewed as an asset of utmost value for all service providers (Andreassen \& Lindestad, 1998).

Measuring customer loyalty, however, is not an easy task (Bauman, Burton \& Eliot, 2005). The complexity in measuring customer loyalty rises from the fact that the concept of loyalty is comprised of behavioral and attitudinal dimensions (Rauyruen \& Miller, 2007). Behavioral loyalty is present if the customer repeats purchases regularly over a period of time. As such, behavioral loyalty is often seen as a mixture of the customer's intention to repeat purchases of services/ products - including frequency and amount (Lewis \& Soureli, 2006). On the other hand, attitudinal loyalty is viewed as consisting of customers' nature, preferences, trust or emotional relatedness to services/products and word-of-mouth (Zeithaml, Berry \& Parasuraman, 1996, Andreassen \& Lindestad, 1998). Yet, it is worth noting that the proposition that attitude and behavior comprise the two most significant components of loyalty has been criticized in marketing literature as well (Bloemer, Ruyter \& Peeters, 1998).

\section{Service quality}

The quality of delivered services certainly represents one of the major "triggers" of loyalty among customers and, as such, - has generated a considerable amount of interest among academics and practitioners. As a term, quality is used commonly in everyday life and we all do have an excellent notion of what represents a good or poor quality. In spite of such a widespread use, interestingly enough, researchers have not come up with a single definition of quality yet, mainly because quality as a concept is related to a great number of interpretations (Garvin, 1984). Indeed, what may be perceived as a superb quality by one individual may also be observed as an average, or even below average, quality by another person. Namely, elements including person's habits, attitudes, experiences, set of values or social class or cultural background are just some of the 
factors determining the way in which quality may be perceived by an individual. This clearly suggests that measuring quality is a composite and challenging task. The complexity of measuring quality is further deepened by some characteristics of services including intangibility and the fact that customers are often involved in the service process, hence directly influencing the quality of the service itself.

True complexity behind measuring quality in service setting is probably illustrated best by Seth, Deshmukh \& Vrat (2005), who, in their study, presented 19 different models used to measure the quality of service. The technical and functional model (Grönroos, 1984), the gap model (Parasuruman, Zeithaml \& Berry, 1985), the performance-only model (Cronin \& Taylor, 1992) and the hierarchical model (Brady \& Cronin, 2001) certainly represent the most prominent models frequently used in modern-day studies. One of the most frequently used models for measuring service quality is the SERVQUAL model (Parasuraman, Zeithaml \& Berry, 1988), which we used for designing the survey instrument for this study.

\section{CRM in contemporary banking}

The fierce competition in the domain of financial services has forced many banks to accept an approach based on the Customer Relationship Management (CRM) concept. Today, banks are oriented towards tight cooperation with their clients, because their survival and further progress often implies delivering a superior value - which should exceed customers' expectations over a longer period of time. In addition, a superior value cannot easily be copied by the competition, which also explains why it represents a corner stone in creating a sustainable competitive advantage. In rather dynamic market conditions, it is not enough for banks to be focused only on creating good quality products and services; also, it is of essential importance to build strong relationships with clients. Basically, the CRM concept puts clients into the spotlight of the overall bank's activity. However, in order to do so, it is important to convert transactions into interpersonal relations, yielding the CRM concept as an essential and unavoidable component of modern bank marketing. On the other hand, the implementation of the "one- to-one marketing" concept is a strategy enabling the development of long-term relationships between a bank and its every individual client. This represents a special challenge particularly for large and financially strong banks, which also have an enormous number of clients.

The implementation of CRM is significant, considering that studies show that users of banks' services are becoming more dynamic and less loyal. Thanks to the Internet, clients have a greater opportunity than ever before to switch their business from one bank to another (Gupta \& Shukla, 2002). The relationship between a bank and its clients is not only a simple sum of processed transactions in the past (Parasuraman, Zeithaml \& Berry, 1988). The strong connectedness between the two sides within the financial services sector is, above all, based on mutual long-term benefits. In that context, banks plan on generating maximum profits through increasing levels of satisfaction and loyalty among clients. On the other hand, by having a long-term cooperation with a given bank, clients expect to be approved more favorable bank loans, an improved quality of the service, as well as to obtain a higher level of confidence in the bank (Binks \& Ennew, 1997). Accepting the marketing orientation is a banks' answer to the impact of various factors from the business environment. It is essential that banks get to know their clients in order to assess risk when approving loans or doing some other transaction. As suggested by Buttle (2004), executing a marketing concept accordingly can assure that banks not only attract new clients, but also retain the existing ones. Truly marketing oriented banks have current and future needs of their clients in the focus of their everyday business. Creating active relationships and close ties with clients is a prerequisite for generating clients' loyalty and banks' long-term profitability.

The management of many banks give advantage and greater importance to corporate clients in comparison to individual clients. Although, fewer in number, corporate clients have more complex financial needs. Individual clients usually choose a bank based on savings interest rates, the affordability of various credit lines, the available range of payment cards offered by a bank and so on. In this domain, smaller banks have many difficulties to match offers made 
by large banks. Unlike markets for individual users, within business-to-business (B2B) markets, the advantages of small banks come to surface far more easily. Regardless of the fact that smaller banks may not have a capacity to completely cover the financial needs of large enterprises, they can successfully meet the needs of micro and small enterprises. Respecting and understanding the client represents a powerful tool for generating long-term cooperation between small banks and micro/small enterprises. Finding an adequate business niche is a foundation for improving the competitive position of a small bank. However, in order to do so, a small bank needs to fulfill important conditions - the orientation towards clients and access to the modern information and communications technology (ICT).

A number of factors have a critical effect on the relationship between banks and corporate clients. These factors include the level of competition in the banking sector, the size of the enterprise, a need for getting a credit or use another bank's service, the transaction costs and financial condition of an enterprise. Generally, large enterprises have a more pronounced need for developing a relationship with a greater number of banks when compared to micro and small enterprises. Also, an enterprise's size sometimes influences its negotiating position. Large enterprises are less dependent on a relationship they create with a given bank, taking into consideration that they have more opportunities to cooperate with other financial institutions as well. On the contrary, as Zineldin (1996) stresses, small enterprises have a greater motive to establish a good, lasting relationship with one bank which they will remain loyal to.

The expansion of ICT allowed the development of electronic banking (e-banking). Transactions are no longer based solely on personal ties and contacts, but also on electronic relationships supported by ICT. This led to performing transactions faster and with fewer mistakes. The following data points to a notable increase in the use of e-banking services. It is estimated that in 2000 some 34.4 million people used the Internet for performing banking transactions. By 2004, the number of e-banking users grew to 122.3 million. The residents of Finland are a typical example of how e-banking can create the added value. According to the OECD data, in April 2005, approximately 2.6 million Finnish residents (representing one half of the entire country's population) used e-banking services (Maenpaa, Kale \& Mesiranta, 2008). Only 3\% of Finnish residents go to a bank branch to make payments or complete some other transaction. Nevertheless, even though the use of CRM cannot be imagined without relying on modern ICT, managers should not undermine the significance of personal liaisons because they represent the foundation of a long-term relationship. Above all, banks must show that they understand and respect their clients, care about them and are sincerely oriented towards solving their problems and financial needs.

\section{SMEs as corporate clients in the banking sector}

The constant rendering of an excellent service quality to customers is extremely significant in all highly competitive sectors, including the banking sector. In their study, Wang, Lo \& Hui, (2003) found that Chinese banks which delivered a superb service quality to their corporate clients had earned a reputation of excellence that ultimately led to higher retention levels among corporate clients. Another spill-over effect was that satisfied corporate clients were less eager to engage in split-banking, i.e. doing business with one or more competitor banks at the time. Studies on loyalty in the banking industry have quite frequently been conducted in marketing literature (Lewis \& Soureli, 2006; Berg, 2008). In fact, many studies on loyalty and factors leading to loyalty in the services sector actually evolved around surveying banks' retail clients (Hallowell, 1996). However, only a few studies have been conducted in relation to understanding loyalty patterns among banks' corporate clients (Lam, Burton \& Lo, 2009), even though they generate a considerable share of revenues to banking institutions (Tyler \& Stanley, 1999).

A number of studies were focused on the factors influencing corporate clients' selection of the bank. Factors related to a range of offered services, service prices, personalized interaction, the affordability of lending and lending rates appeared to be the most significant ones for enterprises when choosing the bank to do business with (Nielsen, Terry \& Trayler, 
1998). The research also suggested that both technical and functional service qualities have a positive effect on the loyalty of SMEs, while stressing that investing in building strong relationships with SMEs will result in improved SMEs' loyalty of to their banks (Madill, Feeney, Riding \& Haines Jr, 2002). On the other hand, Lam \& Burton (2009) were among the first ones to quantify the impact that various variables have on the loyalty of SMEs. Their study found that, in order to maximize loyalty levels among SMEs, banks should devote their efforts to providing an excellent service quality, investing time in nurturing relationships and, especially, developing pricing strategies for loans offered to SMEs.

Finally, in their study, Silver \& Vegholm (2009) addressed the issue related to banks' readiness to adapt to their SME customers. This research found that banks make modest efforts to adapt to their SME clients, mainly due to the banks' centralized organizational structure, lack of communication with SMEs and the insufficient understanding of SMEs' needs. A bank's failure to adapt to the needs of SMEs is, in a sense, furthered when it comes to SMEs' internationalization efforts. Although it has been documented that SMEs are rather dependent on banking/financial institutions when going into foreign markets (Spence, 2003), a recent study by Lindstrad \& Lindbergh (2010) reveals that banks are poorly utilizing their role as a prospective provider of information and services to SMEs going abroad.

\section{RESEARCH METHODOLOGY}

In order to investigate the level of the client satisfaction across different elements of services delivered by banks, an empirical study was conducted through the use of a survey method. The survey method is one of the most frequently employed methods in the marketing field studies. The questionnaire used to gather primary data included 21 statements that mirror various elements of service quality in banking. The respondents expressed their views on a ten-point Likert scale (1 - I completely disagree with the statement; 10 - I completely agree with the statement). The statements were determined based on the review of relevant services marketing literature (Parasuraman, Zeithaml, \& Berry, 1988; Jamal
\& Anastasiadou, 2009). In that manner, we created a foundation for analyzing courtesy, professionalism and competence of employed staff based on the perceptions of clients. In addition, the bank's image as well as the level of empathy shown by the staff towards the client's problems were also analyzed.

The survey involved 133 enterprises whose managers rated the overall service quality of the bank they are doing business with. Those were mainly small and medium enterprises employing up to 250 employees. The majority of corporate clients of the banks that do business in Serbia actually belong to the category of small and medium enterprises. Also, these enterprises are significant in developing the nation's economy. In such a context, the research was directed towards examining attitudes of managers from small and medium enterprises regarding the quality of banking services. Enterprises included in the sample had randomly been chosen from the list of corporate clients that bank had done business with in the previous five years. In addition, all clients were grouped into three segments (large, medium and small clients) on the basis of the annual volume of transactions. The first segment (large clients) included the total of 30 enterprises, the second (medium clients) 48 enterprises, while the third segment (small clients) comprised 55 enterprises. Prior to administering the survey, the questionnaire was pretested on a smaller sample of 15 respondents in order to eliminate possible ambiguities related to the questionnaire. Beside this, in order to create a clear and easy-to-follow questionnaire, researchers had also conducted one group discussion with bank officers.

The data analysis was done in the Statistical Package for the Social Sciences (SPSS). In terms of statistical analysis, we implemented the explorative factor analysis and the variance analysis. The factor analysis was used to group a large number of statements into a smaller number of factors. The reliability of the obtained results and the internal consistency of the statements were measured through the values of the alpha coefficient. The suitability of the data for performing the factor analysis was tested by applying the KaiserMeyer-Olkin (KMO) test and Bartlett's test of sphericity. The testing of evenness among the average values of the statements for the three client groups was done through one-way ANOVA. The use of the mentioned procedure represents a logical solution for comparing 
averages in a situation when there are more than two groups of respondents.

\section{RESEARCH RESULTS}

Prior to doing the factor analysis, the values of the $\mathrm{KMO}$ test $(\mathrm{KMO}=0.877)$ and Bartlett's test $(\mathrm{p}=0.000)$ had shown that the necessary conditions were met for its usage. The values of the KMO index range between 0 and 1 , where the implementation of the factor analysis is inappropriate if the $\mathrm{KMO}$ is lower than 0.5 . Bartlett's test is based on chi-square statistics. The obtained results in our case reveal that we can reject the null hypothesis (the non-existence of a significant correlation between the variables). As a method

Table 1 The results of the explorative factor analysis

\begin{tabular}{|c|c|c|c|}
\hline Statements & Factor 1 & Factor 2 & Factor 3 \\
\hline \multicolumn{4}{|l|}{ Factor 1: Respecting and understanding clients } \\
\hline Paying personal attention to clients & 0,831 & & \\
\hline Courtesy of employed staff & 0,817 & & \\
\hline Respect for clients & 0,798 & & \\
\hline Understanding financial needs of clients & 0,784 & & \\
\hline Focus on what is the best for clients & 0,748 & & \\
\hline Readiness to help clients & 0,741 & & \\
\hline Clients' confidence in the bank's employees & 0,692 & & \\
\hline Orientation towards clients & 0,648 & & \\
\hline Clients' sense of safety & 0,619 & & \\
\hline Providing information to clients & 0,590 & & \\
\hline \multicolumn{4}{|l|}{ Factor 2: Professionalism } \\
\hline Accuracy of the delivered service & & 0,707 & \\
\hline Promptness in providing a service & & 0,694 & \\
\hline Employee appearance & & 0,669 & \\
\hline Efforts made when solving clients' problems & & 0,654 & \\
\hline Respecting previously agreed deadlines & & 0,648 & \\
\hline Fulfilling previously made promises to clients & & 0,558 & \\
\hline \multicolumn{4}{|l|}{ Factor 3: Image } \\
\hline Image with respect to the competition & & & 0,828 \\
\hline Interior & & & 0,820 \\
\hline Modern equipment & & & 0,803 \\
\hline Bank's image & & & 0,663 \\
\hline Branch's image & & & 0,618 \\
\hline Eigenvalue & 6,673 & 3,997 & 3,337 \\
\hline$\%$ of described variance & 31,776 & 19,033 & 15,892 \\
\hline Alpha & 0,94 & 0,82 & 0,84 \\
\hline
\end{tabular}


of the factor analysis, in this research we used the principal component analysis. In order to get a clearer interpretation of the factors, we utilized the varimax rotation.

The results of the factor analysis show that the statements grouped around the three formed factors. The first factor "Respecting and understanding clients" describes $31.776 \%$ of the variance. This factor entails the attributes of the bank's quality of services related to providing personal attention to clients, the courtesy of bank officers, the understanding of clients' financial needs, their readiness to assist clients and so on. The second factor, "Professionalism" describes 19.033\% of the variance. This factor includes the elements of a service such as the precision and promptness of the delivered service, fulfilling promises and respecting the previously agreed deadlines by bank officers. "Image" as the third factor contains elements such as the bank's image, a branch's image, modern equipment and image in comparison to the image of competitors. This factor accounts for $15.892 \%$ of the variance. All three factors describe $66.701 \%$ of the total variance.

All the three factors have a high level of reliability. As it can be seen in Table 1, the values of the alpha coefficient are higher than 0.7, which is the necessary lower threshold of reliability as suggested by Nunnally (1978). Given that high values of the alpha coefficient were obtained - for all the three factors, the values were higher than 0.8 - it can be implied that the statements which grouped around each individual factor are internally consistent.

Finally, by employing the ANOVA test, quite interesting results were obtained with respect to comparing means among corporate clients belonging to different segments (Table 2). Namely, based on the annual volume of transactions that each enterprise conducts through a given bank, three segments were formed: large, medium and small clients. Based on each statement, we wanted to examine if there are statistically significant differences in client perceptions. However, the obtained results stress evenness in attitudes among corporate clients regarding different quality elements of the bank's service offer. Only in the case of the two statements, namely "Efforts made when solving clients' problems" and "Respecting previously agreed deadlines", did differences emerge in the mean
Table 2 The results of the ANOVA test

\begin{tabular}{|c|c|c|}
\hline Statements & $\mathrm{F}$ & Sig \\
\hline Paying personal attention to clients & 0,675 & 0,511 \\
\hline Courtesy of employed staff & 0,543 & 0,582 \\
\hline Respect for clients & 0,910 & 0,405 \\
\hline $\begin{array}{l}\text { Understanding financial needs of } \\
\text { clients }\end{array}$ & 0,841 & 0,434 \\
\hline Focus on what is the best for clients & 0,159 & 0,853 \\
\hline Readiness to help clients & 0,155 & 0,857 \\
\hline $\begin{array}{l}\text { Clients' confidence in the bank's } \\
\text { employees }\end{array}$ & 0,762 & 0,469 \\
\hline Orientation towards clients & 0,733 & 0,482 \\
\hline Clients' sense of safety & 0,243 & 0,785 \\
\hline Providing information to clients & 0,243 & 0,785 \\
\hline Accuracy of the delivered service & 0,928 & 0,398 \\
\hline Promptness in providing a service & 1,596 & 0,207 \\
\hline Employee appearance & 1,049 & 0,353 \\
\hline $\begin{array}{l}\text { Efforts made when solving clients' } \\
\text { problems }\end{array}$ & $2,969^{*}$ & 0,045 \\
\hline $\begin{array}{l}\text { Respecting previously agreed dead- } \\
\text { lines }\end{array}$ & $5,654^{* *}$ & 0,004 \\
\hline $\begin{array}{l}\text { Fulfilling previously made promises to } \\
\text { clients }\end{array}$ & 0,762 & 0,469 \\
\hline Image with respect to the competition & 0,483 & 0,618 \\
\hline Interior & 0,602 & 0,549 \\
\hline Modern equipment & 1,616 & 0,203 \\
\hline Bank's image & 0,016 & 0,984 \\
\hline Branch's image & 0,133 & 0,875 \\
\hline
\end{tabular}

$* * p<0,01 ; \quad * p<0,05$

values of the three observed groups of respondents. In the case of the remaining statements, evenness in mean values is notable in the observed groups.

\section{CONCLUSION}

Contemporary trends in banking business stress the increasing importance of personal interactions between a bank's staff and its clients for the further 
development of their long-term relationships. If a bank wants to strengthen its long-term relationships, it is crucial that an estimate be made on future cash flows the bank will have through cooperation with a given client. However, in order to gain a better perspective regarding the expected events, it is imperative that a bank be fully committed to the marketing orientation.

A good relationship with clients is an important factor not only for improving a bank's competitiveness, but also for meeting business objectives in dynamic market conditions. Creating stronger client relationships should be a daily goal to accomplish through showing care, respect, understanding and empathy for clients, on the one hand, while stressing promptness and accuracy in completing transactions, on the other. Good communication with the client, competent staff and modern ICT certainly represent the three key elements of a bank's marketing strategy. However, without an adequate support of the financial sector, marketing alone cannot help a bank to be doing business profitably in the long-run. Managers must be aware of the fact that clients in retail banking are mostly driven by savings interest rates or the attractiveness of loans when choosing a bank, while corporate clients choose a bank primarily based on a bank's capacity to meet their financial needs.

The findings of our empirical study reveal the significance of the three elements of a bank's service offerings. These are: respect for clients, the professionalism of a bank's staff and its image. The first two factors reflect intangible effects of a bank's service offers and stress the significance of improving interactions with clients, including respect, understanding clients' problems, empathy as well as the prompt and accurate completion of financial transactions. Image, as the third identified factor, also includes the tangible elements of quality such as modern equipment and the appearance of interior in a bank's branches. The results of the conducted study are also implicative of the fact that the bank whose corporate clients were surveyed was able to establish similar levels of the relationship quality with different clients, regardless of the value and frequency of transactions they complete with the bank. Namely, statistically significant differences in clients' responses appeared only in the case of the two observed attributes.
It is important to mention that this study was mainly directed towards measuring the quality of services in corporate banking. It would be useful for future studies to include an analysis of financial elements, such as, the interest rate, the diversity of the offered payment cards, the availability and affordability of loans and home mortgages. It would also be interesting to investigate if, and to what extent, the mentioned elements are important to the client when selecting a bank in comparison to intangible service components. In addition, it would be quite useful to conduct a comparative analysis of attitudes and perceptions amongst a bank's corporate and retail clients.

Future studies could also explore the impact that the factors of service offering have on client loyalty. In that respect, it is useful to design and test a new model that would include three independent variables (respecting and understanding clients, professionalism and image) and one dependable variable (client loyalty). Through employing the multiple regression analysis, the statistical significance of the mentioned factors on client loyalty could be determined. Furthermore, by implementing a moderated regression, apart from the main effects, the interaction effects could be tested as well. In that way, a more universal analysis could be performed on the key loyalty antecedents in corporate banking.

\section{ACKNOWLEDGEMENTS}

This research is a part of the interdisciplinary scientific project (no. 41010) financed by the Ministry of Science, Republic of Serbia.

\section{REFERENCE}

Andreassen, T., \& Lindestad, B. (1998). Customer loyalty and complex services. International Journal of Service Industry Management, 9 (1), 7-23.

Ayyagari, M., Beck, T., \& Demirgüç-Kunt, A. (2007). Small and medium enterprises across the globe. Small Business Economics, 29 (4), 415-434. 
Berg, L. (2008). Loyalty, naivety and powerlessness among Norwegian retail bank customers. International Journal of Consumer Studies, 32 (3), 222-232.

Binks, M. R., \& Ennew, C. T. (1997). The Relationship Between UK Banks and Their Small Business Customers. Small Business Economics, 9 (2), 167-178.

Bloemer, J., Ruyter, K., \& Peeters, P. (1998). Investigating drivers of bank loyalty: the complex relationship between image, service quality and satisfaction. International Journal of Bank Marketing, 16 (7), 276-286.

Brady, M. K., \& Cronin Jr, J. J. (2001). Some new thoughts on conceptualizing perceived service quality: a hierarchical approach. Journal of Marketing, 65 (3), 34-49.

Buttle, F. (2004). Customer Relationship Management. Amsterdam: Elsevier.

Connolly, B. J. (2000). Banks must manage small business deposits to foster long-term growth. Commercial Lending Review, 15 (4), 8-14.

Cronin, J. J., \& Taylor, A. S. (1992). Measuring service quality: a reexamination and an extension. Journal of Marketing, 56 (3), 55-67.

Cunningham, R. M. (1956). Brand loyalty - what, where, how much. Harvard Business Review, 39 (11/12), 116-138.

Evropska Komisija, Generalni direktorat za preduzetništvo $\mathrm{i}$ industriju (2010). European SMEs under pressure: annual report on EU small and medium-sized enterprises 2009. Brussels: European Commission.

Garvin, D. (1984). What does 'product quality' really mean? Sloan Management Review, 26 (1), 25-43.

Grönroos, C. (1984). A service quality model and its marketing implications. European Journal of Marketing, 18 (4), 244-249.

Gupta, M. P., \& Shukla, S. (2002). Learning from Customer Relationship Management Implementation in a Bank. Global Business Review, 3 (1), 99-122.

Hallowell, R. (1996). The relationships of customer satisfaction, customer loyalty, and profitability: an empirical study. International Journal of Service Industry Management, 7 (4), 2742.

Jamal, A., \& Anastasiadou, K. (2009). Investigating the effects of service quality dimensions and expertise on loyalty. European Journal of Marketing, 43 (3/4), 398-420.

Lam, R., Burton, S., \& Lo, H.-P. (2009). Customer tradeoffs between key determinants of SME banking loyalty. International Journal of Bank Marketing, 27 (6), 428-445.
Lewis, B. R., \& Soureli, M. (2006). The antecedents of consumer loyalty in retail banking. Journal of Consumer Behaviour, 5 (1), 15-31.

Lindstrand, A., \& Lindbergh, J. (2011). SMEs' dependency on banks during international expansion. International Journal of Bank Marketing, 29 (1), 65-83.

Madill, J.J., Feeney, L., Riding, A., \& Haines Jr, G. H. (2002). Determinants of SME owners' satisfaction with their banking relationships: a Canadian study. International Journal of Bank Marketing, 20 (2), 86-98.

Maenpaa, K., Kale, S., Kuusela, H., \& Mesiranta, N. (2008). Consumer perceptions of Internet banking in Finland: The moderating role of familiarity. Journal of Retailing and Consumer Services, 15 (4), 266-276.

Nielsen, J.F., Terry, C., \& Trayler, R.M. (1998). Business banking in Australia: a comparison of expectations. International Journal of Bank Marketing, 16 (6), 253-263.

Nunnally, J.C. (1978). Introduction to psychological measurement. New York: McGraw-Hill.

OEBS (2009). The impact of the global crisis on SME and entrepreneurship financing and policy response. Paris: OECD.

Parasuraman, A., Zeithaml, V. A., \& Berry, L. L. (1985). A conceptual model of service quality and its implications for future research. Journal of Marketing, 49 (4), 41-50.

Parasuraman, A., Zeithaml, V. A., \& Berry, L. L. (1988). SERVQUAL: a multiple-item scale for measuring consumer perception of service quality. Journal of Retailing, 64 (1), 1240.

Rauyruen, P., \& Miller, K. E. (2007). Relationship quality as a predictor of B2B customer loyalty. Journal of Business Research, 60 (1), 21-31.

Republički zavod za statistiku (2008). Enterprises in Republic of Serbia according to size: vol. 61, Belgrade: Statistical Office (in Serbian). Available: http://webrsz.stat.gov.rs/axd/ dokumenti/rdokument. 16. nov. 2008

Seth, N., Deshmukh, S. G., \& Vrat, P. (2005). Service quality models: a review. International Journal of Quality and Reliability Management, 22 (9), 913-949.

Shen, Y., Shen, M., Xu, Z., \& Bai, Y. (2009). Bank size and small and medium-sized enterprise (SME) lending: evidence from China. World Development, 37 (4), 800-811.

Silver, L., \& Vegholm, F. (2009). The dyadic bank-SME relationship: customer adoption in interaction, role and 
organization. Journal of Small Business and Enterprise Development, 16 (4), 615-627.

Spence, M. (2003). International strategy formation in small Canadian high-technology companies - a case study approach. Journal of International Entrepreneurship, 1 (3), 27796.

Torre, A. de la, Martínez Pería, M. S., \& Schmukler, S. L. (2010). Bank involvement with SMEs: beyond relationship lending. Journal of Banking and Finance, 34 (9), 2280-2293.

Tyler, K., \& Stanley, E. (1999). Marketing financial services to businesses: a critical review and research agenda. International Journal of Bank Marketing, 17 (3), 98-115.
Wang, Y., Lo, H.P., \& Hui, Y.V. (2003). The antecedents of service quality and product quality and their influences on bank reputation: evidence from the banking industry in China. Managing Service Quality, 13 (1), 72-83.

Zeithaml, V. A., Berry, L. L., \& Parasuraman, A. (1996). The behavioral consequences of service quality. Journal of Marketing, 60 (2), 31-46.

Zineldin, M. (1996). Bank-corporate client "partnership" relationship: benefits and life cycle. International Journal of Bank Marketing, 14 (3), 14-22.

Received 15 April 2012, after one revision, accepted for publication 27 April 2012

Veljko Marinkovic is an assistant professor of Consumer Behavior and Marketing Research the Faculty of Economics, University of Kragujevac, Serbia. He holds Ph.D. in Business Management from the Faculty of Economics, University of Belgrade, Serbia. His major interests are related to customer satisfaction and loyalty measurement, customer profitability analysis, financial services marketing, consumer ethnocentrism.

Vladimir Senic is an assistant professor at the Faculty of Hotel Management and Tourism, University of Kragujevac, Serbia, where he teaches Services Management and Marketing Management courses. He earned his Ph.D. in Economics from the Faculty of Economics, University of Kragujevac, Serbia. The scope of his scientific work and interest is related to issues in the domain of management and marketing of service-based organizations. 